\title{
Quantified Us: a group-in-the-loop approach to team network reconstruction
}

\author{
Raphael Tackx* \\ raphael.tackx@cri-paris.org \\ Université de Paris \\ INSERM U1284, Center for Research \\ and Interdisciplinarity (CRI), F-75006 \\ Paris, France
}

\author{
Leo Blondel ${ }^{*}$ \\ lblondel@alumni.harvard.edu \\ Université de Paris \\ INSERM U1284, Center for Research \\ and Interdisciplinarity (CRI), F-75006 \\ Paris, France
}

\author{
Marc Santolini ${ }^{\dagger}$ \\ marc.santolini@cri-paris.org \\ Université de Paris \\ INSERM U1284, Center for Research \\ and Interdisciplinarity (CRI), F-75006 \\ Paris, France
}

\begin{abstract}
Understanding team collaboration processes is key for the development of new technologies towards increasing groups effectiveness. In particular, the need and challenges of coordinating potentially large-scale, self-organized, collaborative initiatives have been made even more salient by the COVID-19 pandemic. Key to this challenge is the difficulty for participants to situate themselves within the larger social context. At the individual level, the Quantified-Self movement has showcased how insights from one's own data can trigger changes in behavior, sometimes leading to fundamental insights through self-research. Building on these premises, here we present CoSo (Collaborative Sonar), a digital platform for participatory collective sensing and social research. CoSo is a web and mobile data collection platform for team network reconstruction and visualization. It leverages a "group-in-the-loop" intrinsic motivator to collect data on collaborative activities performed by a team through a mobile app, with summary statistics and visualizations of the collected data made available on a web dashboard. We showcase its use and discuss the perspectives offered by promoting grouplevel metacognition and collective introspection. By highlighting the invisible relationships in a group, CoSo facilitates in-the-wild studies, remote/hybrid data collection, and multi-modal sensing approaches of group interactions within professional and other social contexts.
\end{abstract}

\section{CCS CONCEPTS}

- Software and its engineering $\rightarrow$ Software libraries and repositories; • Information systems $\rightarrow$ Information systems applications; Open source software; Retrieval on mobile devices; - Human-centered computing $\rightarrow$ Scientific visualization.

\section{KEYWORDS}

Social networks, Collective sensing, Team interactions, Collaborations, Group-in-the-loop

\footnotetext{
${ }^{*}$ Both authors contributed equally to this work.

${ }^{\dagger}$ To whom correspondence should be addressed: marc.santolini@cri-paris.org
}

Permission to make digital or hard copies of part or all of this work for personal or classroom use is granted without fee provided that copies are not made or distributed for profit or commercial advantage and that copies bear this notice and the full citation on the first page. Copyrights for third-party components of this work must be honored For all other uses, contact the owner/author(s).

UbiComp-ISWC '21 Adjunct, September 21-26, 2021, Virtual, USA

(C) 2021 Copyright held by the owner/author(s)

ACM ISBN 978-1-4503-8461-2/21/09.

https://doi.org/10.1145/3460418.3479363
ACM Reference Format:

Raphael Tackx, Leo Blondel, and Marc Santolini. 2021. Quantified Us: a group-in-the-loop approach to team network reconstruction. In Adjunct Proceedings of the 2021 ACM International foint Conference on Pervasive and Ubiquitous Computing and Proceedings of the 2021 ACM International Symposium on Wearable Computers (UbiComp-ISWC '21 Adjunct), September 21-26, 2021, Virtual, USA. ACM, New York, NY, USA, 6 pages. https://doi. org $/ 10.1145 / 3460418.3479363$

\section{INTRODUCTION}

Understanding how team processes underlie team performance is key for the design of organizational strategies as well as the development of new technologies for making groups more effective. The formation of teams to solve complex problems is salient in Science and Engineering, with over 90 percent of all publications now written by multiple authors [17]. In addition, the past decades have seen the rise of large and open communities of contributors, collaborating and competing to solve problems in ways that traditional organizations are ill structured to manage $[9,16,18]$. Such collaborations introduce unique challenges, from communication to coordination, which, if left unaddressed, can jeopardize the success of the projects.

Prompted by the prevalence of the phenomenon, a series of studies have explored how team composition [12, 27, 30], organisation [1,15] or dynamics [22] determine the performance and survival of teams, usually relying on conceptual models [1] or proxies from scientific co-authorship data [31] to quantify team impact and resilience. For example, team composition and its relation to team success has been measured in collaborative coding in Github [15], in the artistic setup of Broadway musicals [12] or in private organizations $[20,28]$. Complementary to such data-driven and modeling approaches, sociological approaches have provided indepth qualitative insights from case studies, for example through the inspection of laboratory notebooks as anthropological artefacts, revealing the multiple factors that underlie the process through which a group of individuals work together [29].

Yet, we are still lacking the ability to obtain fine-grained, largescale in situ qualitative and quantitative insights on micro-level team processes. The problem comes in part from the fact that human activities, human dynamics, inter/intra collaborations and team organization $[3,8,10,19]$ require the ability to reach individuals to access such information. Data gleaned through ex-post surveys or interviews are typically small in both sample size and in cross-sectional or short time scales-limiting the ability to provide 
generalizable insights to understand the processes and dynamic patterns that underlie team work and performance.

Poised to tackle this problem, the use of smartphones and wearables has been on the rise to derive fine-grained, controlled insights on social interactions. For example, Radio-frequency identification (RFID) badges have been used to map temporal face-to-face group interaction dynamics [2], with numerous real-world applications in human and animal groups. Such an approach yields quantitative insights but requires local human guidance and supervision to ensure a proper usage of the RFID badges, creating a bottleneck for scalability. Smartphone apps on iOS and Android [5] have also been used to derive interaction networks using Bluetooth scanning. Yet, recent legislation on the use of Bluetooth for contact-tracing studies in iOS has limited such attempts to authorized governmental applications. Moreover, such studies focus on face-to-face interactions, limiting the full understanding of social interactions at play. Beyond physical interactions, passively obtained personal data from social platforms and mobile phone data allows for the collection of large interaction datasets [10], generating insights on friendship networks with a statistical power previously unattainable. However, such data is obtained in a non-controlled manner, and the resulting interactions remain hard to qualitatively assess.

To enhance such quantitative insights, recent studies in health and psychiatry $[14,24]$ have leveraged digital mobile applications that use active methods of experience sampling, including explicit self-reports that may range from occasional and detailed survey instruments to more frequent, brief and in-the-moment questionnaires that are referred to as "ecological momentary assessment" (EMA). EMA offers a number of major benefits over traditional survey instruments including the reduction of retrospective bias, realtime tracking of dynamic processes, simultaneous integration of multi-level data, characterization of context-specific relationships, inclusion of interactive feedback, and enhanced generalizability of results [25].

Such active methods leveraging self-report rely on a regular user engagement. Traditional models reward engagement through monetary prizes, making them hard to scale or be replicated in absence of funding. To tackle this issue, the Open Humans platform has implemented a "Quantified-self" participatory system where study participants share their data in an anonymized manner with researchers, who in exchange provide digital notebooks for participants to investigate their own data along with general statistics over the entire cohort [11]. Such an approach has proved to promote regular, long-term engagement in symptom self-report studies during the COVID19 pandemic ("Quantified Flu" project [26]), demonstrating the benefits of including participants in the loop of the experimental research study.

Building on these insights, we present a new web and mobile data collection platform for team network reconstruction called CoSo (Collaborative Sonar). CoSo focuses on a group in the loop intrinsic motivator (Fig. 1) to collect data on activities performed by a team through a mobile app, and present summary statistics and visualizations of the collected data on a companion web dashboard. In the following, we describe the features of the platform, showcase its use on a case study, and discuss the perspectives offered by promoting group-level metacognition and collective introspection.

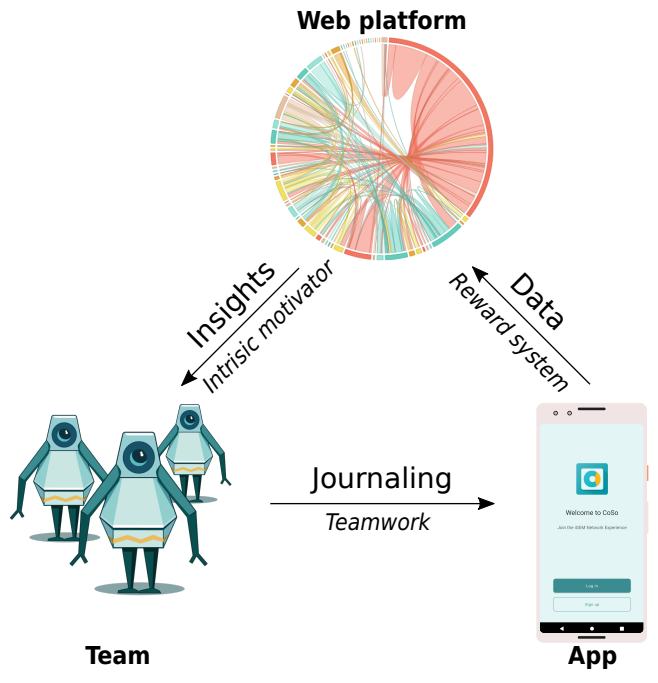

Figure 1: Group-in-the-loop methodology. Team members use the CoSo app to $\log$ activities and collaborations. The web platform then provides immediate insights into the global team structure and dynamics, creating an incentive for engagement and recruitment of peers for further completeness of the visualization reports. As more users participate, the value of the collected data increases for both the research team and the participants. This cycle creates an intrinsic motivator to increase participation in the research project.

\section{CONTEXT}

The design of the CoSo features stems from the study of team-ofteams ecosystems in challenge-based open innovation settings, in particular the iGEM (International Genetically Engineered Machine) synthetic biology competition [6] and the CROWD4SDG citizen science project [21]. In these multi-level settings, participants collaborate within teams, and teams collaborate with one another to solve complex research projects. As such, the features presented below include the annotation of intra- and inter-group interactions, and the proposed tasks represent key activities undergone by the teams during the conduct of their research project.

\section{DESIGN}

CoSo is composed of three main applications. First, a backend gathers all user data and information, and provides an API. Second, a frontend web application allows for data visualization, team management, filling surveys and receiving notifications. Finally, a mobile application for Android and iOS allows users to journal tasks, receive push notifications, fill surveys and gather immediate insights about their logged data. The researchers running the study have a special administrative access on the front end to create surveys and communications to be sent to all or some participating teams. The open-source code is made available here: https://gitlab.com/interactiondatalab/coso. 

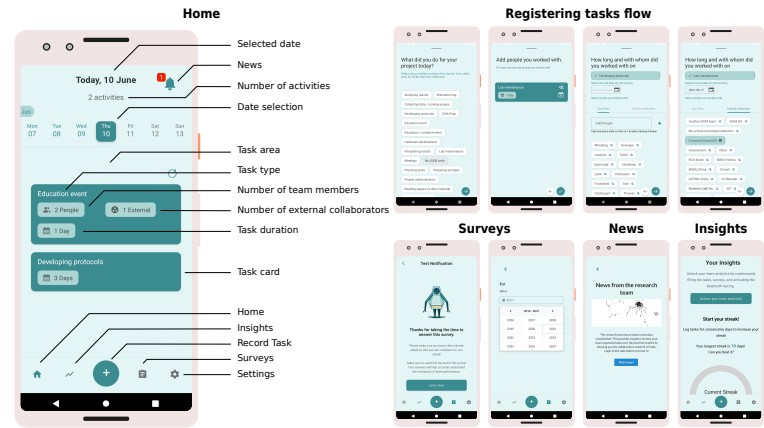

Figure 2: Presentation of the CoSo app and its main flows. The Home screen allows for activity reporting and is the main entry point towards all other screens. The users see the activities registered for a particular day and can edit the information contained within them. On the top is a bell icon displaying the number of unread "News" (communications by the research team). At the bottom are buttons to navigate between the different screens: the Home, the Insights tab containing analytics, the Surveys screen allowing the user to answer custom surveys by the research team, and the Settings screen. Finally, prominently located at the center is the button to launch an Activity Registration (or activity recording). This button leads to the activity registration flow displayed on the right. The user is presented with several types of actions and can select any number of them. Then, for each task, the user is invited to add team collaborators and external collaborators, as well as select an end date for the task.

\subsection{Data collection}

First we discuss the data collection mechanisms allowing the research team to gather information from team members.

3.1.1 Granular task journaling. The key feature of the CoSo app is the ability for individuals within a team to record their activities and collaborations. To facilitate engagement, this is the main action a user can take on the home page (Fig. 2). Upon registering an activity, a user is presented with a selection of possible activities as defined by the research team. In addition, the user can create a custom activity within a predefined activity category that will then appear within the team activity list, allowing for a customisation beyond the set of predefined categories from the research team. Once the activities have been selected, the user can select for each of them the team members who participated in them, as well as broad external collaborations types if relevant, for example interteam collaborations in a team-of-teams context. Finally, the user can set a duration (start and end date) for the task. Overall, these features aim at facilitating the collection of temporal data on the task allocation structure and external collaborations of a team.

3.1.2 Long-form surveys. In addition to regular activity reporting, the research team can create through the web interface custom surveys and submit them to a subset or all of the registered teams. The surveys are presented to the team members on both the web interface and in the application within the survey screen. We implemented common survey fields such as custom text or radio button

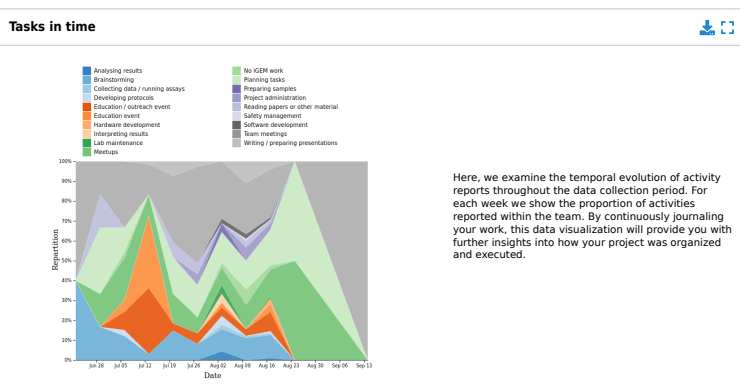

Figure 3: Example data visualization from the web platform. By registering activities and plugging external services, the users can unlock data visualizations on the web platform. These data visualizations are crafted by the research team to provide insights into the team collaborative work. Each card has a title, a descriptive text, as well as an interactive data visualization. The latter can be downloaded as an SVG to be easily included in the team external communication.

matrices, as well as unique internal selectors such as a field that allows the user to select other team members. This allows the research team to collect both individual-level information (e.g role in the team, demographics, background), as well as relational data (friendships, perception of roles within the team, close collaborators). The collected data can be exported as a JSON file through an API to allow the research team to create custom data visualizations.

3.1.3 External tools. In addition to the self-reported data collected from the app, we implemented in the web interface the ability to connect to OAuth based external services. This allows for the passive gathering of data from other communication channels the team is using. For example, in the context of iGEM we collect user edits on the team digital lab notebook on the iGEM MediaWiki instance. In addition, we implemented a connector to Slack, such that metrics about team communications can be extracted.

3.1.4 Notification system. In order to increase user engagement with the app, we implemented a notification system that regularly reminds the users to record their activity. We chose to send a reminder every week on Sundays if the user did not record any activity during the past week. Finally, social studies often require direct communication between the research team and the participants. In CoSo, the research team can create a news item from the web interface, and send it to either the whole user base, or a particular team. This news item contains an image, a free form text and a call to action button with a custom link and a custom text. The bell icon at the top of the home screen carries a red bubble with the number of unread news. Upon interaction it takes the user to a specific screen which contains all received news. This offers opportunities for the research team to quickly communicate with teams involved in the study in a distributed manner.

\subsection{Incentivization through meta-cognition}

Here, we discuss the "group-in-the-loop" features for team members to obtain a direct feedback on the collected data. 
3.2.1 Visualization of individual-level and team-level data. To incentivize the data collection process, CoSo provides participants with a visualization dashboard of the collected data. The web interface contains interactive data visualization cards with an export function (Fig. 3). These data visualizations represent the records of team activity on the app (activity journaling) and on the external services. In particular, the ability to obtain data visualizations by plugging external services containing pre-existing data lowers the cost of entry to the CoSo app, highlighting the benefits of further self-report through the app to refine the insights on team dynamics. In addition, we implemented gamification features within the mobile application. The Insight tab within the app shows the user their current and longest streak (number of consecutive days with an activity recorded), along with various user-level and team-level metrics: the total number of activities recorded by the user, the five most frequent activity categories recorded by the user, their top five collaborators, the number of teams in the study, the total number of tasks and collaborations recorded, and finally the five users with the longest streak.

3.2.2 Team management. Each team has access to a team management section on the web interface to visualize who is registered in the study and who is using the mobile app. Furthermore, due to the fluid nature of studied teams, we added the possibility for the team to add a user who was not initially registered in the database, allowing to ensure proper enrollment in the study. This new user can then be selected in the activity reporting, surveys and subsequent data visualization filtering.

\section{CASE STUDY}

Here, we present the results of a pilot study involving one team participating in the 2020 iGEM competition, using an early version of the app over 50 days (Fig. 4a). In this version, users could only report ego-centered activities (i.e only activities in which they participated), and the web dashboard was not implemented. Out of the 20 student members actively participating in the iGEM competition, 19 used the app. Activity reports were collected using the CoSo app. The dataset obtained consisted of the activity category, the user ID who created the activity report, the timestamp and the list of teammates reported as collaborators in the activity.

We find that the most reported activities involve a large number of team members: Team meetings, Meetups, Education / Outreach event, Planning tasks and Brainstorming. This might reflect the fact that collective events promote the use of the app, creating an over-representation of these activities. This might also reflect the fact that team coordinators are more involved in the data collection process. Indeed, when looking at user recording (Fig. 4b), we can see that only a small fraction of the team members recorded these activities. This suggests that some team members were assigned the role of journaling the activities for the entire team. In the current version of the app, we allow for a non-ego-centered approach where only one or a few team members can annotate interactions that they did not participate in. The aim is to leverage team gatherings as a time for a team reporter to collect team activities and interactions, with an incentive for completeness provided by the visualization feedback mechanism.

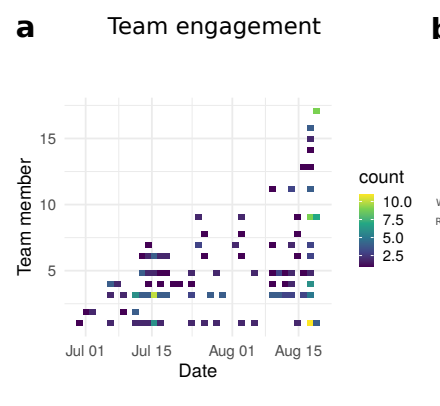

b Journaling of the activity

C
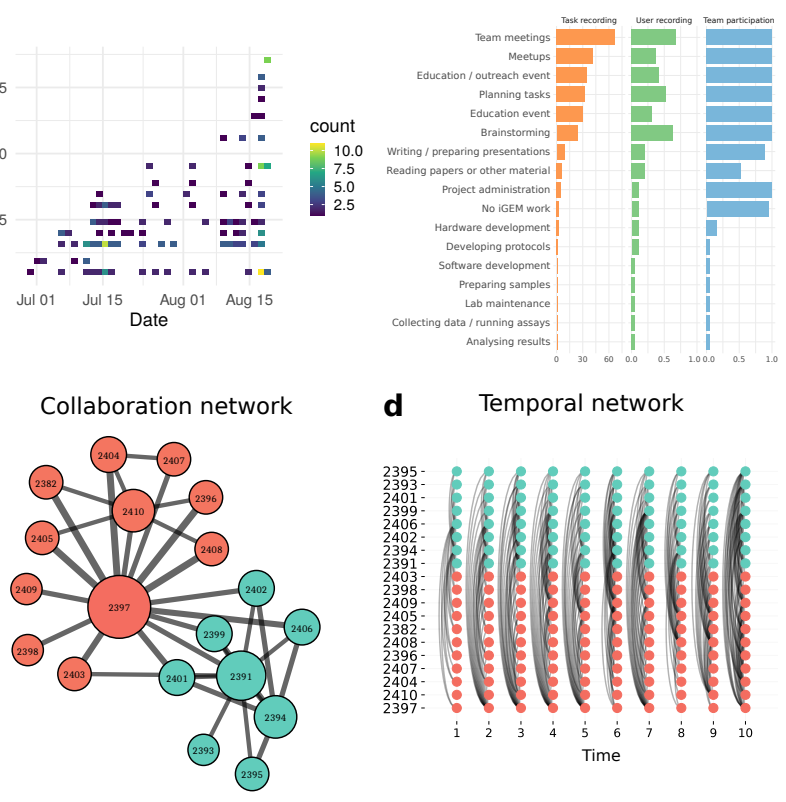

d Temporal network

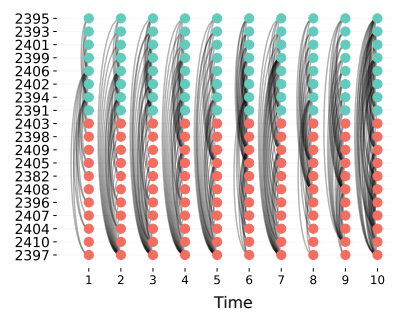

Figure 4: Example data generated with the CoSo app. a) User engagement over time. Lines correspond to users and are ordered chronologically by the date of first report. Squares indicate activities, colored by the number of reports in that period. While 19 users have declared an activity, some users display a stronger and more regular engagement, with $\sim 30 \%$ of users contributing $80 \%$ of the reports. b) Summary statistics of the activity reports: number of times an activity category was reported, proportion of team members who reported a category, and proportion of members who were mentioned as collaborators in a category. c) Collaboration network between users. Links depict collaborations reported at least 5 times. d) Evolution of the team network across 10 consecutive time windows in the study. Colors in c, $d$ correspond to topological communities detected in c using the modularity algorithm [4].

The collaborations recorded within activities can be represented as a bipartite network and projected into the user space by counting the number of activities within which two users participate (Fig. 4c). This yields a weighted collaboration network that reveals two subteams using community detection [4]. Finally, the temporal network in Fig 4d illustrates the mutual participations in activities between team members over ten consecutive time windows spanning the data collection period, highlighting changes in collaboration structure and intensity over time.

\section{DISCUSSION}

In this study we introduce the CoSo platform for team interaction studies. Compared to other app-based survey collection softwares $[13,14]$, CoSo is designed to be deployed across a large number of teams with a focus on gathering intra- and inter-team interactions 
through activity reports and surveys on team interactions. Moreover, CoSo uses gamification strategies within the app and data visualizations to promote engagement. These could be extended with visualizations and leaderboards providing social comparison to further incentivize active usage. Beyond the particular setting of team studies, this technology can be used to record general interactions between individuals identifying as a group, and allow them to gather insights on the group overall structure and dynamics that might be invisible to the individuals involved. Several future perspectives are foreseen for the extension to such cases. First, a white labeling of the app is intended to provide the ability for any group to sign up as a team and facilitate group introspection. This updated version will be implemented within upcoming studies on open innovation collaborative ecosystems. A limitation of this approach is that it could be used in a professional setting to peer-pressure employees to sign-up for monitoring their activities, though such behavior would be non-compliant with GDPR regulations. The ability to choose varying levels of anonymization (in particular within the visualizations) could be considered, to allow both for a radical openness (interest in individual roles within a group) or for complete anonymity of the visualization obtained (interest in overall group structure and diagnostics of potential bottlenecks). In particular, solutions leveraging trustless encryption technologies similar to blockchain healthcare record management systems [23] could be implemented. The open-source nature of the platform and the block architecture of visualizations both promote the local usage of the app and the implementation of custom visual elements. Similarly, the "external apps" section allows to include a variety of OAuth based services and use the CoSo platform as an insight hub. Finally, the app can be modified to access sensors such as Bluetooth, Wi-Fi or GPS from mobile devices as well as wearables, allowing for further usage in a "quantified us" setting appropriate for blended experiences. While an early version of the app implemented Bluetooth sensing [7], novel regulations for contact tracing apps have prevented us from deploying this feature on iOS platforms. We note that contact tracing studies could still be implemented in the context of Android-based studies, enhancing CoSo for hybrid data collection of group interactions.

\section{ACKNOWLEDGMENTS}

We thank Lionel Deveaux, Romain Delory, Elmar Tavares, Radhika Beaumé and Hugo Laplace for their involvement in the technological design and implementation, Savandara Besse for her help with setting the case study, and Rathin Jeyaram and Robert Ward for their insights on the general design of the app and visualizations. Thanks to the Bettencourt Schueller Foundation long term partnership, this work was partly supported by the CRI Research Fellowship to Marc Santolini. This work was further supported by the European Union's Horizon 2020 research and innovation programme under grant agreement No 872944. Ethical approval for the case study was granted by the IRB00003888.

\section{REFERENCES}

[1] Daniel Barkoczi and Mirta Galesic. 2016. Social learning strategies modify the effect of network structure on group performance. Nat. Commun. 7 (Oct. 2016), 13109.

[2] Alain Barrat, Ciro Cattuto, Vittoria Colizza, Jean-Francois Pinton, Wouter Van den Broeck, and Alessandro Vespignani. 2008. High resolution dynamical mapping of social interactions with active RFID. (Nov. 2008). arXiv:0811.4170 [cs.CY]

[3] Vincent D Blondel, Adeline Decuyper, and Gautier Krings. 2015. A survey of results on mobile phone datasets analysis. EPJ Data Science 4, 1 (Aug. 2015), 10.

[4] Vincent D Blondel, Jean-Loup Guillaume, Renaud Lambiotte, and Etienne Lefebvre. 2008. Fast unfolding of communities in large networks. F. Stat. Mech. 2008, 10 (Oct. 2008), P10008.

[5] Tjeerd W Boonstra, Mark E Larsen, and Helen Christensen. 2015. Mapping dynamic social networks in real life using participants' own smartphones. Heliyon 1, 3 (Nov. 2015), e00037.

[6] J Brown. 2007. The iGEM competition: building with biology. IET Synth. Biol. 1, 1 (June 2007), 3-6.

[7] Mathieu Cunche, Antoine Boutet, Claude Castelluccia, Cédric Lauradoux, and Vincent Roca. 2020. On using Bluetooth-Low-Energy for contact tracing. Research Report. Inria Grenoble Rhône-Alpes ; INSA de Lyon. https://hal.inria.fr/hal02878346

[8] Pierre Deville, Dashun Wang, Roberta Sinatra, Chaoming Song, Vincent D Blondel, and Albert-László Barabási. 2014. Career on the move: geography, stratification, and scientific impact. Sci. Rep. 4 (April 2014), 4770.

[9] Chiara Franzoni and Henry Sauermann. 2014. Crowd science: The organization of scientific research in open collaborative projects. Res. Policy 43, 1 (Feb. 2014), $1-20$.

[10] Fosca Giannotti, Luca Pappalardo, Dino Pedreschi, and Dashun Wang. 2013. A Complexity Science Perspective on Human Mobility.

[11] Bastian Greshake Tzovaras, Misha Angrist, Kevin Arvai, Mairi Dulaney, Vero Estrada-Galiñanes, Beau Gunderson, Tim Head, Dana Lewis, Oded Nov, Orit Shaer, Athina Tzovara, Jason Bobe, and Mad Price Ball. 2019. Open Humans: A platform for participant-centered research and personal data exploration. Gigascience 8, 6 (June 2019).

[12] R Guimera. 2005. Team Assembly Mechanisms Determine Collaboration Network Structure and Team Performance. , 697-702 pages.

[13] Saurabh Gupta, Mandeep S Dhillon, Siddhartha Sharma, Riddhi Gohil, and Sunny Sachdeva. 2021. Epicollect5: A Free, Fully Customizable Mobile-based Application for Data Collection in Clinical Research. , 248-251 pages.

[14] Arno Klein, Jon Clucas, Anirudh Krishnakumar, Satrajit S Ghosh, Wil Van Auken, Benjamin Thonet, Ihor Sabram, Nino Acuna, Anisha Keshavan, Henry Rossiter, Yao Xiao, Sergey Semenuta, Alessandra Badioli, Kseniia Konishcheva, Sanu Ann Abraham, Lindsay M Alexander, Kathleen R Merikangas, Joel Swendsen, Ariel B Lindner, and Michael P Milham. 2020. Remote Digital Psychiatry: MindLogger for Mobile Mental Health Assessment and Therapy. (Nov. 2020), 2020.11.16.385880 pages.

[15] Michael Klug and James P Bagrow. 2016. Understanding the group dynamics and success of teams. R Soc Open Sci 3, 4 (April 2016), 160007.

[16] Camille M Masselot, Bastian Greshake Tzovaras, Chris L B Graham, Gary Finnegan, Rathin Jeyaram, Isabelle Vitali, Thomas E Landrain, and Marc Santolini. 2021. Co-Immune: a case study on open innovation for vaccination hesitancy and access. medRxiv (2021).

[17] Staša Milojević. 2014. Principles of scientific research team formation and evolution. Proc. Natl. Acad. Sci. U. S. A. 111, 11 (March 2014), 3984-3989.

[18] Michael Nielsen. 2011. Reinventing Discovery.

[19] J G Oliveira and A-L Barabási. 2005. Human Dynamics: The Correspondence Patterns of Darwin and Einstein. (Nov. 2005). arXiv:physics/0511006 [physics.socph]

[20] Pentland, A. 2012. The new science of building great teams. Harv. Bus. Rev. 90, 4 (2012), 60-69.

[21] Barbara Pernici. 2020. CROWD4SDG: Crowdsourcing for sustainable developments goals. Pearson, Upper Saddle River, NJ. 248-252 pages.

[22] Christoph Riedl and Anita Williams Woolley. 2017. Teams vs. Crowds: A Field Test of the Relative Contribution of Incentives, Member Ability, and Emergent Collaboration to Crowd-Based Problem Solving Performance. AMD 3, 4 (Dec. 2017), 382-403.

[23] Shuyun Shi, Debiao He, Li Li, Neeraj Kumar, Muhammad Khurram Khan, and Kim-Kwang Raymond Choo. 2020. Applications of blockchain in ensuring the security and privacy of electronic health record systems: A survey. Computers \& Security 97 (Oct. 2020), 101966. https://doi.org/10.1016/j.cose.2020.101966

[24] John Torous, Mathew V Kiang, Jeanette Lorme, and Jukka-Pekka Onnela. 2016. New Tools for New Research in Psychiatry: A Scalable and Customizable Platform to Empower Data Driven Smartphone Research. JMIR Ment Health 3, 2 (May 2016), e16.

[25] Timothy J Trull and Ulrich W Ebner-Priemer. 2020. Ambulatory assessment in psychopathology research: A review of recommended reporting guidelines and current practices. , 56-63 pages.

[26] Bastian Greshake Tzovaras, Enric Senabre Hidalgo, Karolina Alexiou, Lukasz Baldy, Basile Morane, Ilona Bussod, Melvin Fribourg, Katarzyna Wac, Gary Wolf, and Mad Ball. 2021. Quantified Flu: an individual-centered approach to gaining sickness-related insights from wearable data. medRxiv (2021).

[27] Brian Uzzi, Stefan Wuchty, Jarrett Spiro, and Benjamin F Jones. 2012. Scientific teams and networks change the face of knowledge creation. In Networks in Social Policy Problems. Cambridge University Press, 47-59. 
[28] Duncan Watts. 2016. The organizational spectroscope. Medium, April 1 (2016).

[29] Steve Woolgar and Bruno Latour. 1986. Laboratory life: the construction of scientific facts. Princeton University Press.

[30] Anita Williams Woolley, Christopher F Chabris, Alex Pentland, Nada Hashmi, and Thomas W Malone. 2010. Evidence for a collective intelligence factor in the performance of human groups. Science 330, 6004 (Oct. 2010), 686-688.

[31] Stefan Wuchty, Benjamin F Jones, and Brian Uzzi. 2007. The increasing dominance of teams in production of knowledge. Science 316, 5827 (May 2007), 1036-1039. 